"This is an Accepted Manuscript of an article published by Society of Plastics Engineers, Wiley, in Polymer Engineering and Science, on July 3, 2015, available online:

https://doi.org/10.1002/pen.24133 


\title{
CONTINUOUS MODIFICATION OF
}

\section{POLYPROPYLENE VIA PHOTOINITIATION}

\author{
Yasaman Amintowlieh, Costas Tzoganakis*, and Alexander Penlidis \\ Institute for Polymer Research, Department of Chemical Engineering, University of \\ Waterloo, Waterloo, Ontario, N2L 3G1, Canada
}

*Author to whom correspondence should be addressed (costas.tzoganakis@uwaterloo.ca)

\begin{abstract}
A twin screw extruder was used for continuous modification of polypropylene (PP) via UV radiation. Long chain branches were incorporated in the PP backbone to modify its rheological properties. Benzophenone $(\mathrm{BPH})$ as photoinitiator and trimethylolpropane triacrylate (TMPTA) as coagent were utilized during PP photomodification. Radiation was carried out after mixing in the extruder on solid stretched strands with approximately $0.3 \mathrm{~mm}$ thickness. The effects of photoinitiator concentration, radiation time and coagent presence were studied via a replicated two-level full factorial design of experiments. It was shown that photomodification of PP can be done continuously. Formation of long chain branches (LCBs) in the experimental runs was confirmed via rheological measurements. Gel content of the samples was also measured. It was found that long chain branches can be formed in PP with and without TMPTA at certain processing conditions. The amount of gel in the samples prepared with TMPTA was higher; however, the gel content could be controlled by manipulating $\mathrm{BPH}$ concentration and radiation time.
\end{abstract}




\section{INTRODUCTION}

Extruders have long been used as continuous reactors for polypropylene (PP) chemical modification [1-4]. This process is known as reactive extrusion (REX) and it has been employed to produce controlled rheology PP (CRPP) [5,6]. In order to produce CRPP via REX, PP and peroxides are fed into the extruder, and initiation reactions followed by $\beta$ scission of the PP chains take place during melting and mixing in the extruder. These reactions are responsible for degradation of PP. As soon as temperature reaches the peroxide decomposition temperature, the peroxide abstracts hydrogens from the PP backbones and macroradicals are thus formed. Since PP tertiary radicals are unstable, the chain will break ( $\beta$-scission) and polymer with lower molecular weight (MW) and narrower molecular weight distribution (MWD) will be formed [6].

Utilizing thermo-chemical initiators, such as peroxides, in REX has its own disadvantages, namely, limited controllability. Peroxides reach their decomposition temperature prior to effective mixing with PP, thus causing an excessive and non-homogenous degradation in PP. In order to overcome this issue, photoinitiators were used along with UV irradiation to efficiently degrade PP. In this way, the reaction initiation step and subsequent formation of macroradicals become independent of the processing temperature and the reaction only starts when UV irradiates the PP/photoinitiator mixture. He et al. [7] used this technique to modify PP rheology during extrusion. Photomodification was conducted in the last two zones of the extruder by opening the barrel and exposing the mixture to UV irradiation.

Photoinitiators were not only used to produce CRPP, but also to modify the melt strength of PP or other polyolefins by incorporating long chain branches (LCBs) to their structure [811]. Increasing PP melt strength is possible by introducing LCBs to the PP structure. Producing long chain branched PP (LCBPP) is more challenging than CRPP, since $\beta$-scission reactions should be controlled by stabilizing PP radical centers. This is not trivial, since $\beta$ scission reactions are dominant at temperatures above $60^{\circ} \mathrm{C}[12]$. The typical temperature for PP processing is well above $60{ }^{\circ} \mathrm{C}$ ( $\mathrm{T}_{\text {process }}>160{ }^{\circ} \mathrm{C}$, the $\mathrm{PP}$ melting point); thus, excessive degradation is inevitable. In order to overcome this issue, and make sure that the modification 
was indeed feasible, the radiation was first carried out in the batch mode and solid state [13]. Processing conditions that essentially "preserve" macroradicals were determined by manipulating the processing variables. The processing variables, including photoinitiator concentration, duration of irradiation, UV lamp intensity, process temperature, and photoinitiator type, were optimized to encourage radical combinations rather that $\beta$-scission. The effects of different combinations of these variables on rheological properties, molecular weight characteristics and branching levels were studied in the solid state. It was found that when the UV lamp intensity was low, BPH concentration was more than $0.3 \mathrm{wt} \%$ (in the total mixture), and radiation was carried out for more than 5 minutes at temperatures below $60{ }^{\circ} \mathrm{C}$, the melt strength of PP was increased significantly. It was found that during the first minute of the modification process, degradation was the dominant reaction. As the radiation duration increased, the frequency of combination reactions increased, and therefore more branches were formed. Thus, LCB and degradation reactions happen simultaneously. Details about these reactions and the mechanisms involved have been discussed in publication [14].

When lower radiation time was needed, trimethylolpropane triacrylate (TMPTA) was added to $\mathrm{PP} /$ photoinitiator mixture as a coagent to increase the rate of LCB. TMPTA is a trifunctional acrylic monomer, which reacts with the radical centers and prevents them from $\beta$-scission. However, addition of coagent encourages formation of excessive gel in the samples [15], which might not be desirable for certain applications. Hence, processing variables were manipulated to find conditions which resulted in minimum gel content in the samples, while the number of long chain branches was still high. It was found that when the concentration of BPH was high (above $0.5 \mathrm{wt} \%$ ) and the TMPTA concentration low (below $0.2 \mathrm{wt} \%$ ), branching content was sufficiently large, while gel content could be controlled below $5 \%$. All these modifications (producing LCBPP via UV radiation) were successfully conducted after mixing PP with the photoinitiator in the batch mode in solid state $[13,14]$.

However, since LCBPP has numerous commercial applications, there is a need to find a method to continuously modify PP and scale up the system. He et al. [16] irradiated a $\mathrm{PP} /$ photoinitiator $(\mathrm{BPH}) /$ pentaerythritol triacrylate (PETA) mixture during melt mixing in the extruder by removing the barrel (REX). They found that the modified PP had better 
foamability due to greater melt strength compared to the parent PP. PETA was used as a crosslinking agent along with BPH to generate long chain branched PP. However, possible drawbacks of UV radiation in the extruder include limited UV penetration depth into the thick plastic melt and excessive degradation due to high processing temperatures. As mentioned before, the latter issue makes formation of long chain branches possible only if coagents (like PETA) are used to "block" $\beta$-scission reactions.

In this work, the target was to introduce a continuous processing method for modification of PP via UV-irradiation. Modification was conducted in the solid state, and a twin screw extruder was used to continuously supply polypropylene for the modification. Although continuous photomodification of PP was inspired from REX, UV modification was carried out after the strands exited the die and solidified. Formation of long chain branches (LCBs) in PP was assessed with and without the aid of a coagent as a radical stabilizer. Trimethylolpropane triacrylate (TMPTA), a trifunctional acrylic coagent was used to "block" chain scission. The extruded solid strands were stretched to increase UV radiation penetration depth by decreasing strand thickness. Also, it is speculated that chain orientation, which happens due to external extensional forces, decreases degradation. Rheological measurements were used to evaluate the effect of $\mathrm{BPH}$ concentration, radiation time and coagent presence on viscoelastic properties and gel content. The (optimal) combination of these variables that resulted in long chain branching (LCB) rather than degradation or crosslinking (CL) was found.

\section{MATERIALS AND METHODS}

\section{MATERIALS}

Polypropylene homopolymer (PP2255E1) from ExxonMobil (USA) with melt flow rate (MFR) of $3.5 \mathrm{~g} / 10 \mathrm{~min}\left(230{ }^{\circ} \mathrm{C}, 2.16 \mathrm{~kg}\right)$ was used as the parent PP. Benzophenone (BPH), $99 \%$ purity, was purchased from Sigma-Aldrich (Oakville, ON, Canada) and used as 
photoinitiator. Trimethylolpropane triacrylate (TMPTA) was also purchased from SigmaAldrich and used as coagent. Irganox 1010, Ciba Specialty Chemicals (now BASF, Germany), was used as antioxidant to prevent PP thermal degradation.

\section{DESIGN OF EXPERIMENTS}

In order to study the effect of photoinitiator concentration, radiation time and coagent presence on the continuous modification of PP, a replicated two-level full factorial design was used (see Table 1). Runs 1 to 8 represent the $2^{3}$ factorial design and run 9 is the centerpoint, which was independently replicated (run 10). Runs 11 and 12 were conducted in addition to the $2^{3}$ factorial design (and its centerpoints) in order to generate more information about the process. Finally, run 13 is PP mixed with 1 wt\% BPH, which has only been passed through the extruder with no UV radiation. The properties of this run are shown for the sake of comparison. Viscoelastic properties along with the gel content of the runs were measured and statistically analyzed via Design-Expert 8.0.7.1 software. Runs 9 and 10 were used to obtain an error estimate for further assessment of the significance of mathematical model terms. These models are discussed later in section 3.

Table 1 shows the different levels of the actual and coded variables (factors). The coded levels (the last three columns) are used in the models of section 3.1.1. 
Table 1: Experiments for $2^{3}$ full factorial design (with centerpoint replicates)

\begin{tabular}{ccccccc}
\hline Run \# & $\begin{array}{c}\text { BPH-A } \\
(\mathbf{w t} \%)\end{array}$ & $\begin{array}{c}\text { Time-B } \\
(\mathbf{s})\end{array}$ & $\begin{array}{c}\text { Coagent-C } \\
(\mathbf{w t} \%)\end{array}$ & $\mathbf{A}(\mathbf{w t} \%)$ & $\mathbf{B}(\mathbf{w t} \%)$ & $\mathbf{C}(\mathbf{s})$ \\
\hline 1 & 0.5 & 16 & 0 & -1 & -1 & -1 \\
\hline 2 & 1 & 16 & 0 & 1 & -1 & -1 \\
\hline 3 & 0.5 & 36 & 0 & -1 & 1 & -1 \\
\hline 4 & 1 & 36 & 0 & 1 & 1 & -1 \\
\hline 5 & 0.5 & 16 & 0.25 & -1 & -1 & 1 \\
\hline 6 & 1 & 16 & 0.25 & 1 & -1 & 1 \\
\hline 7 & 0.5 & 36 & 0.25 & -1 & 1 & 1 \\
\hline 8 & 1 & 36 & 0.25 & 1 & 1 & 1 \\
\hline 9 & 0.75 & 26 & 0.13 & 0 & 0 & 0 \\
\hline 10 & 0.75 & 26 & 0.13 & 0 & 0 & 0 \\
\hline 11 & 0.75 & 26 & 0 & 0 & 0 & -1 \\
\hline 12 & 0.75 & 26 & 0.25 & 0 & 0 & 1 \\
\hline 13 & 1 & 0 & 0 & - & - & - \\
\hline Parent PP & - & - & - & - & - & - \\
\hline
\end{tabular}

\section{Preparation METHOD}

In order to improve mixing of photoinitiator and PP in the twin screw extruder, a PP/BPH master batch was prepared prior to extrusion. PP with $7 \mathrm{wt} \% \mathrm{BPH}$ were melt-mixed in a batch mixer at $190{ }^{\circ} \mathrm{C}$ and $80 \mathrm{rpm}$ for 8 minutes. The same processing conditions were used for preparing the TMPTA and polypropylene master batch ( $2 \mathrm{wt} \%$ TMPTA). The mixtures were subsequently ground using a Wiley mill (Model 1102, Arthur H. Thomas Co.). After grinding, the master batches were diluted with PP to prepare the recipes of the runs in Table 1. The final mixing was carried out in a Leistritz LSM 30.34 co-rotating twin-screw extruder $(\mathrm{L} / \mathrm{D}=28)$ with 8 heating zones (including the die). The temperature set points for these eight zones along with the screw configuration are shown in Figure 1. 


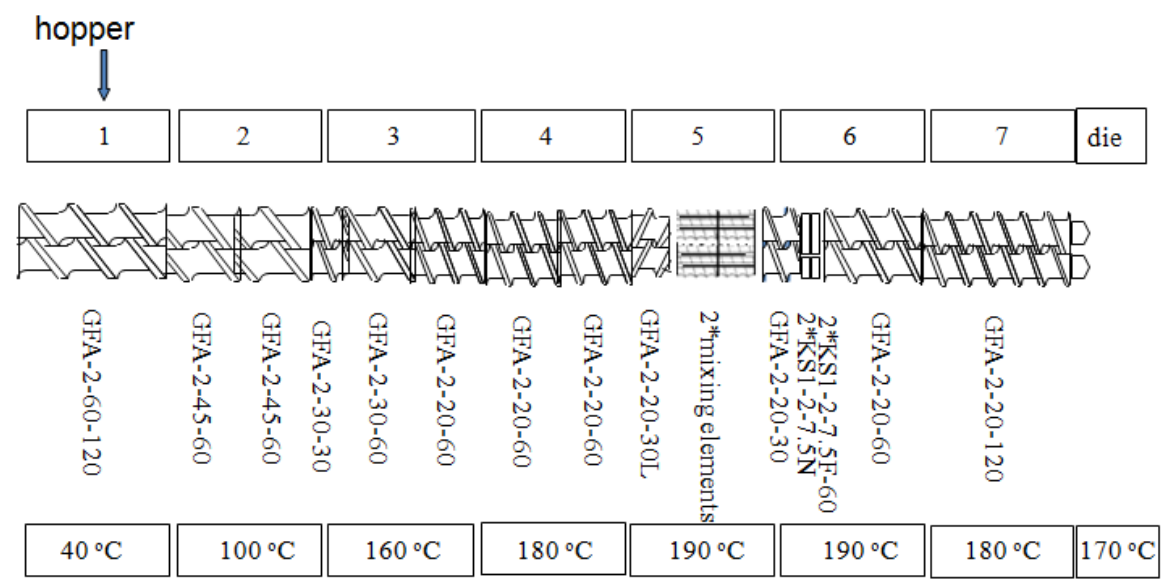

Figure 1: Twin screw extruder screw combination and temperature of the zones

The die diameter was $1.5 \mathrm{~mm}$ and the melted strands were cooled down in a water bath subsequent to their exit from the die. The solidified strands were stretched via two freely rotating rollers (see the schematic of the operation in Figure 2). Irradiation was carried out at this step via a UV Developer Kit from UV Process Supply Inc. (Versa Cure, Chicago, IL, USA). The mercury lamp power was $3.0 \mathrm{~kW}$. The irradiated strands were collected using a winder $(35 \mathrm{rpm})$. The strands reach a diameter of approximately $0.3 \mathrm{~mm}$ after stretching between the rollers due to extensional forces applied by the winder. UV-modified PP was compression-moulded into discs with $25 \mathrm{~mm}$ diameter and $1 \mathrm{~mm}$ thickness at $190{ }^{\circ} \mathrm{C}$ under an applied force of $4,400 \mathrm{~N}$ for five minutes. The discs were used for further characterization tests. 


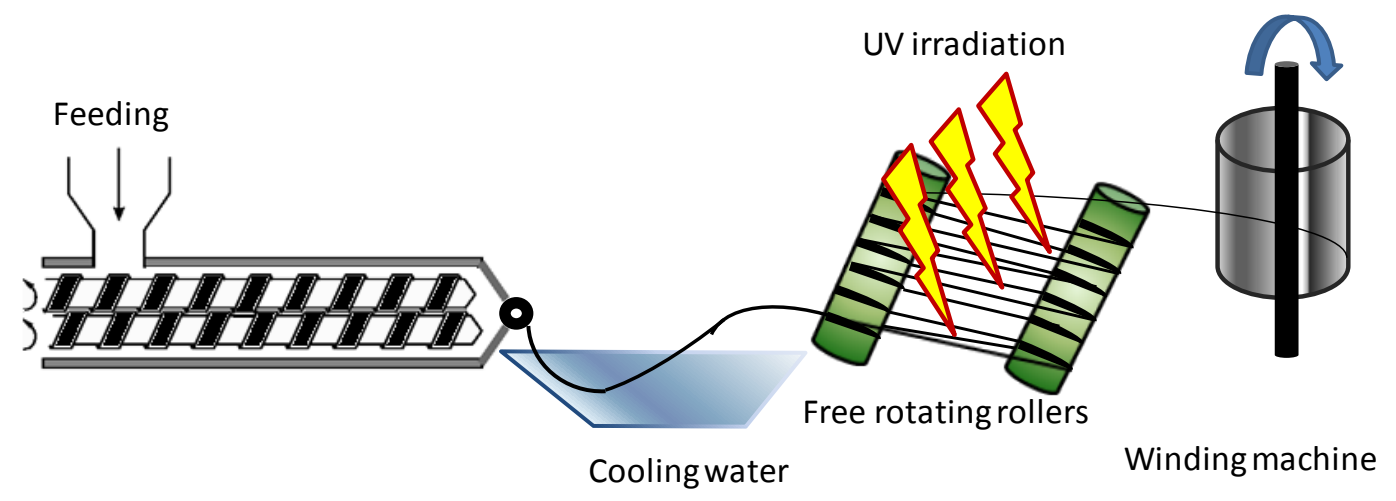

Figure 2: The experimental setup for continuous modification of polypropylene via UV radiation

\section{PARAllel Plate RHEOMETRY}

A stress-controlled parallel plate rheometer (AR2000, TA instruments, New Castle, DE, USA) was used at $190^{\circ} \mathrm{C}$ to measure the viscoelastic properties of the modified PP. Strain sweeps were carried out to identify the linear viscoelastic region during the tests and rheological measurements were carried out at the percentage of strain at which viscoelastic properties remain constant over the whole range of frequencies. Frequency sweeps were subsequently performed in the range of $0.01-100 \mathrm{~Hz}$. Storage modulus ( $\left.\mathrm{G}^{\prime}\right)$, loss modulus $\left(G^{\prime \prime}\right)$, complex modulus $\left(G^{*}\right)$, loss tangent $(\tan (\delta))$, and complex viscosity $\left(\eta^{*}\right)$ were obtained at different angular frequencies $(\omega)$.

The Cross model was fit to $\eta^{*}$ vs. $\omega$ data to estimate the shear thinning index (n), zero shear viscosity $\left(\eta_{0}\right)$ and relaxation time $(\lambda)$ of each sample ((1) using the MATLAB curve fitting toolbox.

$$
\eta^{*}=\frac{\eta_{0}}{1+(\omega \lambda)^{n}}
$$

It is worth noting that $\eta_{0}$ is the limiting value of the complex viscosity at very low shear rates where the viscosity of the polymer melt is independent of shear rate (Newtonian viscosity). $\lambda$ represents the terminal relaxation time whose inverse is related to a characteristic shear rate 
for departure from the Newtonian plateau. $\mathrm{n}$ is the shear thinning index, which is the slope of the shear thinning region in the $\eta^{*}-\omega$ plots.

An increase in the zero shear viscosity ( $\left.\eta_{0}\right)$ of a run compared to the parent PP indicates higher molecular weight, which can be due to the presence of long chain branches. Long chain branched PP (LCBPP) has larger zero shear viscosity $\left(\eta_{0}\right)$ and relaxation time $(\lambda)$, but smaller shear thinning index (n) than linear PP [17].

Rheological polydispersity indices were determined using (2 to (4 [18].

$$
\begin{gathered}
P I=\frac{10^{5}}{G_{c}(\mathrm{~Pa})} \\
\text { ModSep }=\frac{\omega^{\prime}}{\omega^{\prime \prime}} \\
\mathrm{ER}=\mathrm{C}_{1}\left(G^{\prime}{ }_{\text {at } G^{\prime \prime}=500 \mathrm{~Pa}}\right)
\end{gathered}
$$

PI is the so-called rheological "polydispersity index", ModSep stands for "Modulus Separation" and ER is another polydispersity index, more indicative of the high molecular weight end, as introduced by Shroff and Mavridis [18]. These useful rheological polydispersity indices relate to MWD breadth and branching. $G_{c}$ in Equation 2 is the crossover modulus (in Pa), which is the modulus at which $\mathrm{G}^{\prime}$ and $\mathrm{G}^{\prime \prime}$ are equal. In $\left(3, \omega^{\prime}\right.$ and $\omega^{\prime \prime}$ are the angular frequencies when $\mathrm{G}^{\prime}$ and $\mathrm{G}^{\prime \prime}$ are equal to $1000 \mathrm{~Pa}$. Finally, in $\left(4, \mathrm{C}_{1}\right.$ is the slope of the $\log \left(\mathrm{G}^{\prime}\right)$ versus $\log \left(\mathrm{G}^{\prime \prime}\right)$ curve, evaluated within the range of 0.01 to $100 \mathrm{~s}^{-1}$.

In (2 and (3, PI correlates inversely with $\mathrm{G}_{\mathrm{c}}$, and ModSep shows the $\mathrm{G}^{\prime}$ and $\mathrm{G}^{\prime \prime}$ distance from each other at a specific modulus (1000 Pa). It is expected that the values of PI and ModSep reflect the MWD of the runs, while ER is only sensitive to the high MW end of the MWD. When the MWD becomes broader in general, an increase in PI and a decrease in ModSep are expected. However, ER only increases if the distribution of high MW chains broadens due to formation of more high MW chains due to LCB [18]. PI, ModSep and ER were calculated for all of the runs and the results are presented and discussed in detail in section 3. 


\section{GEL PERMEATION CHROMATOGRAPHY (GPC)}

$1 \mathrm{~g}$ of the ground polymer was placed in pouches (sample holder). The pouches were made from stainless steel 120 mesh sheets. They were then sealed and immersed in $100 \mathrm{ml}$ of

xylene at its boiling temperature. $1 \mathrm{~g}$ of Irganox 1010 was added to the solution to prevent degradation of the dissolved PP. The dissolution process was continued for 4 hours and then the stainless steel pouches were taken out of the solution. $20 \mathrm{ml}$ acetone was used as nonsolvent to precipitate the dissolved polymer from the solution. The precipitated polymer was filtered and dried in an oven at $60^{\circ} \mathrm{C}$ for 6 hours. This procedure led to the elimination of gel (insoluble fraction) from the samples to be subsequently analyzed via GPC. Hence, only the sol fraction of each sample was analyzed via GPC.

High temperature GPC (Polymer CHAR, Spain) was used to determine molecular weight (MW), molecular weight distribution (MWD), polydispersity index (PDI) and intrinsic viscosity $[\eta]$ of the samples. Refractive index, Infra-red (IR) and viscometer detectors were employed to characterize each slice of the chromatogram. The GPC set-up had three columns in series (PLgel Olexis mixed), and each column was $30 \mathrm{~cm}$ long with a diameter of $7.5 \mathrm{~mm}$. 1, 2, 4-trichlorobenzene (TCB) was used as the GPC solvent at $135^{\circ} \mathrm{C} .13-15 \mathrm{mg}$ of each sample was dissolved in $9 \mathrm{ml}$ of TCB at $160{ }^{\circ} \mathrm{C}$ for 90 minutes. The solutions were visually inspected first for complete dissolution prior to injection. $250 \mathrm{mg} / \mathrm{l}$ of Irganox 1010 was used as stabilizer.

Information from the differential viscometer, which was one of the GPC detectors, was used to find the intrinsic viscosity $([\eta])$ of each fraction of the samples. The root mean squared radius of gyration $\left(\mathrm{R}_{\mathrm{g}}\right)$ is related to the intrinsic viscosity $[\eta]$ of a polymer of molecular weight $\mathrm{M}$ in solution via (5 [19].

$$
[\eta] \propto \frac{\mathrm{R}_{\mathrm{g}}}{M}
$$

It is known that the presence of LCB leads to lower end-to-end distance of polymer chains and denser polymer coils. Thus, a lower $R_{g}$ and $[\eta]$ will result for a long chain branched 
polymer compared to its linear counterpart of the same molecular weight. Therefore, in a plot of $R_{g}$ or $[\eta]$ versus $M$, the linear samples will show linear behaviour, whereas a branched polymer will exhibit a negative deviation from the linear behaviour (deviation towards smaller $[\eta]$ values) at higher molecular weights. One can use these plots for each slice of the GPC chromatogram, thus creating a contrast between linear and branched structures [20,21].

For a more quantitative measure, the Zimm-Stockmayer branching parameter is used to determine number of branches in the polymer. This parameter represents the ratio of the mean squared radius of gyration of branched to linear polymer. The Zimm-Stockmayer branching parameter, g, can also be obtained from the ratio of intrinsic viscosities of branched to linear polymer ((6) [20].

$$
g=\frac{R_{g_{\text {branch }}}}{R_{\text {g linear }}}=\left(g^{\prime}\right)^{1 / \varepsilon}=\left(\frac{[\eta]_{\text {branch }}}{[\eta]_{\text {linear }}}\right)^{1 / \varepsilon}
$$

In $(6, \varepsilon$ is a constant between $0.5-1.5$ for different branching configurations. Scorah et al. [20] presented theoretical functions for $\varepsilon$ of different branching structures. This value is equal to 0.75 for randomly branched polymers. In $(6, \mathrm{~g}$ becomes lower than 1 for branched PP. Zimm and Stockmayer [22] suggested (7 for the evaluation of the weight average number of long chain branches per molecule $(\mathrm{Bw})$. This equation for a trifunctional randomly branched polymer is [22]:

$$
g=\frac{6}{B w}\left[0.5\left(\frac{B w}{7}\right)^{0.5}+\ln \left(\frac{(2+B w)^{0.5}+B w^{0.5}}{(2+B w)^{0.5}-B w^{0.5}}\right)-1\right]
$$

\section{DETERMINATION OF GEL CONTENT}

The gel content of samples was determined by solvent extraction. The method followed is described in ASTM D2765-11. Approximately $0.3 \mathrm{~g}$ of a ground sample was placed in stainless steel 120 mesh pouches. The pouches were sealed and immersed in $350 \mathrm{ml}$ of boiling xylene. $1 \mathrm{wt} \%$ antioxidant (Irganox 1010) was added to the solvent. After 12 hours, the stainless steel pouches were removed from the boiling solvent and dried out at $120{ }^{\circ} \mathrm{C}$ in 
a drying oven for 12 hours. The measurements were independently replicated at least once for each sample.

\section{RESULTS AND DISCUSSION}

\subsection{RHEOLOGICAL PROPERTIES}

Zero shear viscosity $\left(\eta_{0}\right)$, relaxation time $(\lambda)$, shear thinning index $(n)$, and rheological polydispersity indices (PI, ModSep and ER) of the runs were calculated using (1 to 4. Table 2 summarizes these values for all experimental runs along with the parent PP. The last column in Table 2 (gel content) will be discussed later in section 3.2.

Run 13 is PP mixed with $1 \mathrm{wt} \% \mathrm{BPH}$, which has only been passed through the extruder with no radiation. Comparing the viscoelastic properties of this run with the parent PP (last row) shows that the processing step in the twin screw extruder followed by extension of the solid strand does not significantly affect the viscoelastic properties of the polymer. 
Table 2: Viscoelastic properties $\left(\eta_{0}, \lambda\right.$ and $\left.n\right)$, rheological polydispersity indices (PI, ModSep and ER) and gel content of the experimental runs

\begin{tabular}{cccccccc}
\hline Run \# & $\begin{array}{c}\boldsymbol{\eta}_{\mathbf{0}} \\
\text { (kPa.s) }\end{array}$ & $\begin{array}{c}\boldsymbol{\lambda} \\
(\mathbf{s})\end{array}$ & $\mathbf{n}$ & PI & ModSep & ER & $\begin{array}{c}\text { Gel content } \\
(\boldsymbol{\%})\end{array}$ \\
\hline 1 & 5.5 & 0.8 & 0.52 & 4.0 & 3.5 & 0.18 & 0 \\
\hline 2 & 7.3 & 1.2 & 0.51 & 3.7 & 3.5 & 0.20 & 0 \\
\hline 3 & 6.5 & 2.7 & 0.50 & 6.9 & 2.9 & 0.27 & 0 \\
\hline 4 & 184.6 & 12103.6 & 0.43 & 8.5 & 1.8 & 0.69 & 6.3 \\
\hline 5 & 8.2 & 0.6 & 0.57 & 3.3 & 3.9 & 0.15 & 0 \\
\hline 6 & 81.1 & 848.8 & 0.45 & 11.0 & 1.9 & 0.73 & 2.6 \\
\hline 7 & 984.4 & $107,623.0$ & 0.50 & 16.1 & 1.2 & 0.94 & 16.4 \\
\hline 8 & $1,060.2$ & $110,798.0$ & 0.47 & 11.2 & 1.3 & 1.22 & 12 \\
\hline 9 & 21.6 & 44.2 & 0.46 & 7.4 & 2.3 & 0.42 & 0 \\
\hline 10 & 17.9 & 29.4 & 0.46 & 7.0 & 2.3 & 0.45 & 1.4 \\
\hline 11 & 4.7 & 1.4 & 0.50 & 5.9 & 3.1 & 0.22 & 0 \\
\hline 12 & 6.4 & 7.8 & 0.45 & 8.4 & 2.6 & 0.37 & 1.4 \\
\hline 13 & 9.3 & 0.6 & 0.60 & 3.5 & 3.7 & 0.14 & 0 \\
\hline Parent PP & 10.6 & 0.6 & 0.60 & 3.3 & 3.8 & 0.16 & 0 \\
\hline
\end{tabular}

Data presented in Table 2 show that when the BPH concentration increases, $\eta_{0}, \lambda$ and ER increase and $\mathrm{n}$ decreases. As mentioned in section 2, these changes in $\eta_{0}, \lambda, \mathrm{n}$ and ER reflect formation of LCBs. The reason is higher possibility of hydrogen abstraction from PP chains due to the presence of a larger amount of photoinitiator. Thus, more radical chain combination reactions will happen, which leads to a larger number of LCBs.

In addition, as the radiation time increases, an increase in $\eta_{0}, \lambda$, ER and a decrease in $n$ happen, which again reflect formation of a larger number of LCBs. When radiation time increases, chances for macroradical combination increase, which again leads to formation of more LCBs [14].

Addition of coagent stabilizes PP radical centers and prevents them from degradation. Thus, runs with coagent show larger $\eta_{0}, \lambda$, ER and more shear thinning behaviour (decrease in $\mathrm{n}$ ) compared to the runs prepared with no coagent.

Unlike ER, changes in PI and ModSep do not directly indicate formation of branches and can be due to changes in the breadth of the MWD, which might have happened during UV 
modification. However, analysis of these indices is important in order to identify the effect of modification on the MWD of the polymer. In general, increase in BPH concentration, radiation time, or addition of coagent lead to broadening of the MWD, and consequently, an increase in PI and a decrease in ModSep will result. The main factors and factor interactions which affect PI and ModSep significantly will be discussed shortly in section 3.1.1, along with other response variables ( $\eta_{0}, \lambda, \mathrm{n}$ and ER). It is worth emphasizing that since ER is only affected by the distribution of the high molecular weight chains, it can reflect formation of branches directly.

The above mentioned trends are in agreement with our earlier results for batch modification of PP [13, 23]. However, in previous offline/batch experiments (irradiation of sheets with $1 \mathrm{~mm}$ thickness), no significant change in the properties of the polymer samples was obtained after a few seconds (16-36 s) of radiation. It was found that at least 5 min of radiation time was needed to reach significant LCB levels in these runs. The reason for the significant increase in $\eta_{0}, \lambda$ and ER after low radiation times (see runs 4 and 6 of Table 2) is the low thickness of the radiated strands. It was found that as the sample thickness decreases, the effect of photomodification becomes more pronounced throughout the sample [14]. The other possibility is that chain orientation, which happens during stretching of the PP strands, decreases $\beta$-scission reactions. $\beta$-scission reactions are encouraged by PP chain motions, while these motions are prevented when chains are stretched under extensional forces [12]. In order to study the viscoelastic properties in more detail, $\eta^{*}-\omega$ and $\tan \delta-G^{*}$ are shown in Figure $3 \mathrm{a}$ and $\mathrm{b}$ for runs 1 to 4 (runs without coagent), and in Figure $3 \mathrm{c}$ and $\mathrm{d}$ for runs 5, 6, 7 and 8 (runs with coagent). 

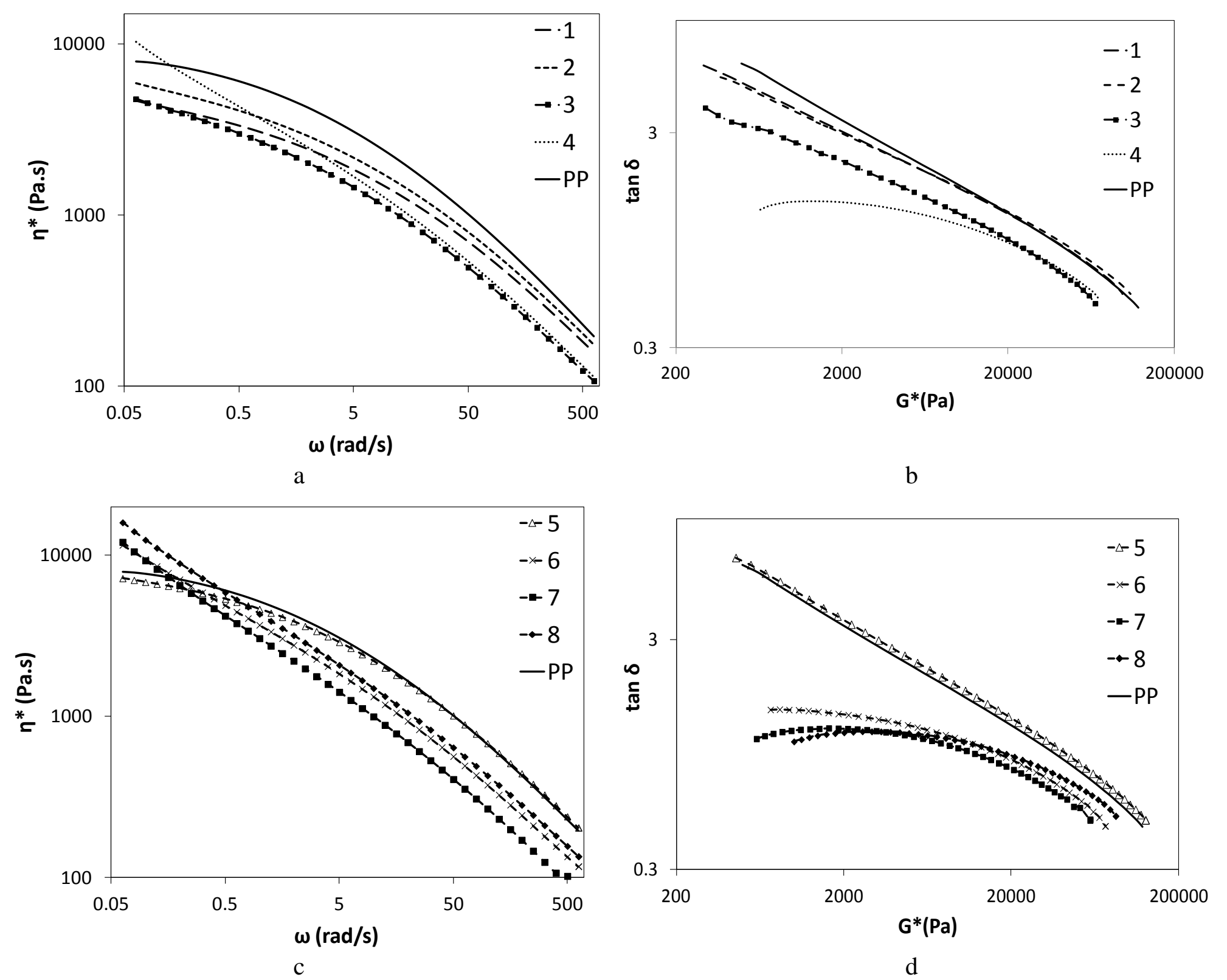

Figure 3: Comparison between viscoelastic properties of the runs without coagent a) $\eta^{*}-\omega$ and b) tan $\delta-G^{*}$, and runs with coagent c) $\left.\eta^{*}-\omega, d\right) \tan \delta-G^{*}$

In Figure $3 a\left(\eta^{*}-\omega\right)$, run 4 has significantly larger $\eta^{*}$ at low frequencies and exhibits more shear thinning behaviour than the parent PP and all other runs. This significant shear thinning behaviour of run 4 resulted in lower viscosity compared to the parent PP at high frequencies, which is an advantage due to easier processing of the modified PP. Moreover, run 4 shows 
larger $\mathrm{G}^{\prime}$ values at low frequencies, which once again verifies formation of long chain branches in this run. G' $\omega$ graphs are not shown herein for the sake of brevity; however, these plots and additional discussion can be found in reference [23]. The slope of the G'- $\omega$ curve in run 4 was also significantly lower than all other runs at low frequencies, which is another indication of formation of branches in this run (LCBPP has a lower G'- $\omega$ slope at low frequencies). Although LCBs were formed in runs 2 and 3 (more shear thinning behaviour along with a shorter plateau region were observed in runs 2 and 3, Figure 3a), the number of long chain branches was not large enough to compensate for the effect of formation of low MW chains. These low MW chains are formed due to $\beta$-scission reactions, which happen in parallel to LCB reactions.

The $\tan \delta-\mathrm{G}^{*}$ plot (Figure $3 \mathrm{~b}$ ) is an indicator of the MWD characteristics of the runs. It is shown that all runs have broader distributions than the parent PP. This is in agreement with the PI and ModSep values of runs 1 to 4, which are cited in Table 2 (larger PI and lower ModSep of runs 1 to 4 compared to the parent PP). Moreover, according to $\tan \delta$-G* plots, the distribution becomes broader in the runs radiated for longer time (runs 3 and 4). As radiation time increases, more macroradical combination reactions can happen and chains with different length will be formed, which causes broadening of the MWD.

Figure $3 c$ and d compare $\eta^{*}-\omega(c)$ and $\tan \delta-G^{*}(d)$ plots of the runs prepared with 0.25 wt $\%$ of coagent (runs 5 to 8). In Figure 3c, all runs, except run 5, have larger $\eta^{*}$ at low frequencies compared to the parent PP, which indicates larger MW of the modified runs. Due to presence of branches in runs 6, 7 and 8, these runs not only show more shear thinning behavior, but also have larger elastic modulus compared to the parent PP $\left(\mathrm{G}^{\prime}-\omega\right.$ plots are not shown again for the sake of brevity). The slopes of the $G^{\prime}-\omega$ curves are significantly lower at low frequencies, which confirms formation of branches in these runs. In Figure 3d, all runs except run 5, which is expected to be degraded, significantly deviate from the parent PP. This shows that the MWD of these modified runs has been significantly changed in comparison to the parent PP. 


\subsubsection{EMPIRICAL MODELS AND TRENDS}

The data points presented in Table 2 were analyzed with Design-Expert (statistical software). Empirical models were fit to each response. These models are cited in Table 3. The significant variables were chosen based on the Shapiro-Wilk test, the significance of the final model and the significance of the lack of fit of the final model (models with minimum lack of fit were favoured). It is worth noting that the lack of fit was insignificant for all models shown in Table 3.

The last row in Table 3 (gel content) will be discussed later in section 3.2.

Table 3: Empirical models for viscoelastic properties and gel content based on coded variables

\begin{tabular}{cc}
\hline Empirical model & Equation \# \\
\hline $1 / \sqrt{ } \eta_{0}=8.15 \mathrm{E}-3-2.41 \mathrm{E}-3 * \mathrm{~A}-2.89 \mathrm{E}-3 * \mathrm{~B}-2.55 \mathrm{E}-3 * \mathrm{C}+1.97 \mathrm{E}-3 * \mathrm{ABC}$ & $(8)$ \\
\hline $\log _{10}(\lambda)=1.79+0.87 * \mathrm{~A}+1.49 * \mathrm{~B}+0.90^{*} \mathrm{C}-0.83 * \mathrm{ABC}$ & $(9)$ \\
\hline $\mathrm{n}=0.49-0.027 * \mathrm{~A}-0.018^{*} \mathrm{~B}+0.019 * \mathrm{ABC}$ & $(10)$ \\
\hline$(\mathrm{ModSep})^{1.45}=3.96-0.84 * \mathrm{~A}-1.51^{*} \mathrm{~B}-0.83 * \mathrm{C}+0.87 * \mathrm{ABC}$ & $(11)$ \\
\hline $1 / \sqrt{\mathrm{ER}}=1.6-0.29 * \mathrm{~A}-0.41^{*} \mathrm{~B}-0.26^{*} \mathrm{C}+0.24 * \mathrm{ABC}$ & $(12)$ \\
\hline $\mathrm{PI}=7.8+2.55 * \mathrm{~B}+2.07 * \mathrm{C}-1.38^{*} \mathrm{AB}-1.79^{*} \mathrm{ABC}$ & $(13)$ \\
\hline $\mathrm{Log}_{10}(\mathrm{Gel}+0.0017)=-1.07+0.83^{*} \mathrm{~A}+1.03 * \mathrm{~B}+1.04 * \mathrm{C}-0.86^{*} \mathrm{ABC}$ & $(14)$ \\
\hline
\end{tabular}

In Table 3, variables $\mathrm{A}, \mathrm{B}$ and $\mathrm{C}$ are $\mathrm{BPH}$ concentration, radiation time and coagent concentration (as per Table 1), respectively. $\mathrm{AB}$ and $\mathrm{ABC}$ denote two factor (BPH concentration-radiation time) and three factor interactions (BPH concentration-radiation time-coagent concentration), respectively. In the equations of Table 3, factors and their interactions are shown in terms of coded variables (see Table 1 for the coded values of these variables). The equations of Table 3 confirm the trends discussed earlier during the analysis of Table 2. Zero shear viscosity $\left(\eta_{0}\right)$ and relaxation time $(\lambda)$ are significantly and positively affected by BPH concentration (A), radiation time (B) and coagent concentration (C). Also, shear thinning index (n) is negatively affected by BPH concentration and radiation time (more shear thinning behaviour due to formation of LCBs). In order to study these factors 
along with their interactions more closely, 3D interaction plots were constructed. Figure 4a and $b$ show the effect of $\mathrm{ABC}$ interaction on $\eta_{0}$ in the absence and presence of coagent, respectively. The other interaction plots for shear thinning index (n) and relaxation time $(\lambda)$ showed the same trends as those for zero shear viscosity $\left(\eta_{0}\right)$. Thus, the interaction plots for these responses are not shown here for the sake of brevity. The maximum of $\eta_{0}, \lambda$ and the minimum of $\mathrm{n}$ are reached when TMPTA coagent was used in the runs. Also, an increase in $\eta_{0}$ and $\lambda$ and a decrease in $\mathrm{n}$ values are more significant at high BPH concentration compared to low $\mathrm{BPH}$ concentration ranges (Figure $4 \mathrm{a}$ and $\mathrm{b}$ ).

(11, (12 and (13 reflect changes in the MWD, when BPH concentaration, radiation time and coagent concentration change. An increase in any of these variables leads to broader MWD with a tail in the high molecular weight ranges (larger ER and PI, and lower ModSep). Using the models shown in Table 3, ABC interactions plots were constructed for the rheological polydispersity indices. Among these rheological indices, only interaction plots for ER are shown in Figure 4c and d (for runs with and without coagent), since the interaction plots for ModSep and PI were in agreemnet with ER. It can be seen that as radiation time increases, ER increases. The reason is broadening of the MWD towards high MW ranges. This is due to formation of long chain branches, which causes formation of a tail in the MWD and broadens the MWD in general. Same as with the other viscoelastic properties, the changes in polydispersity indices are more significant at higher BPH concentrations. 


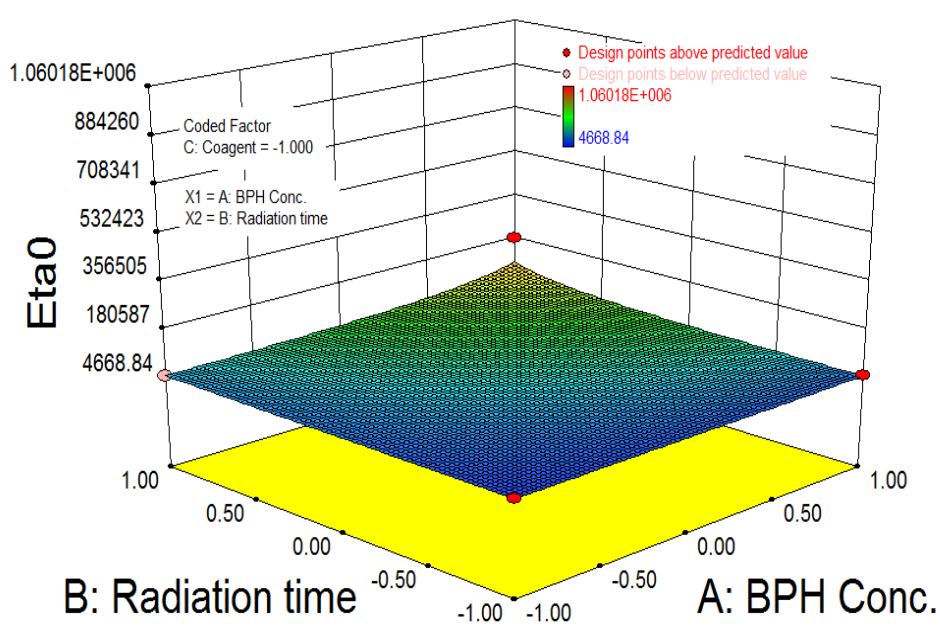

a

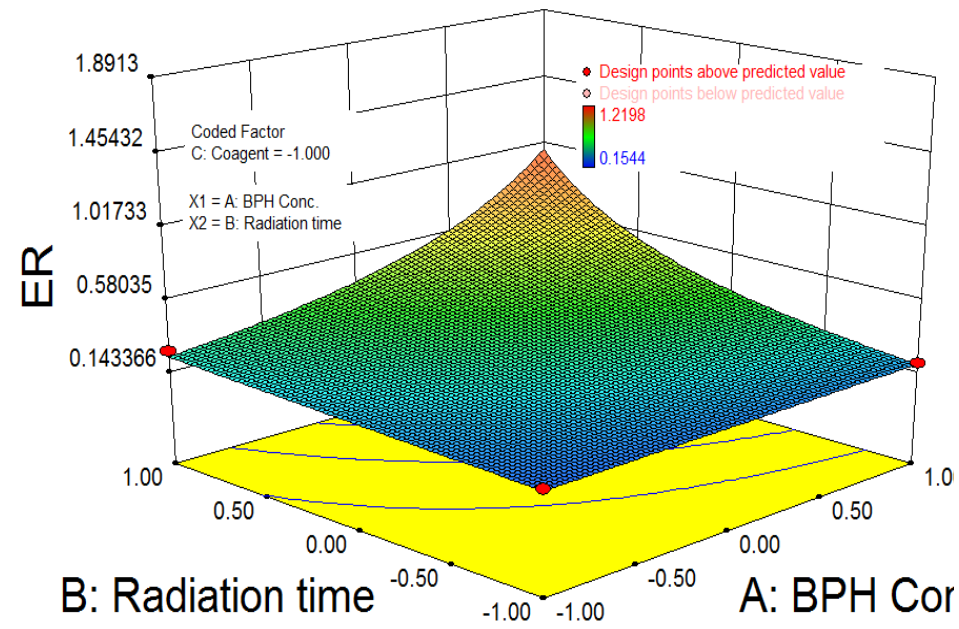

C

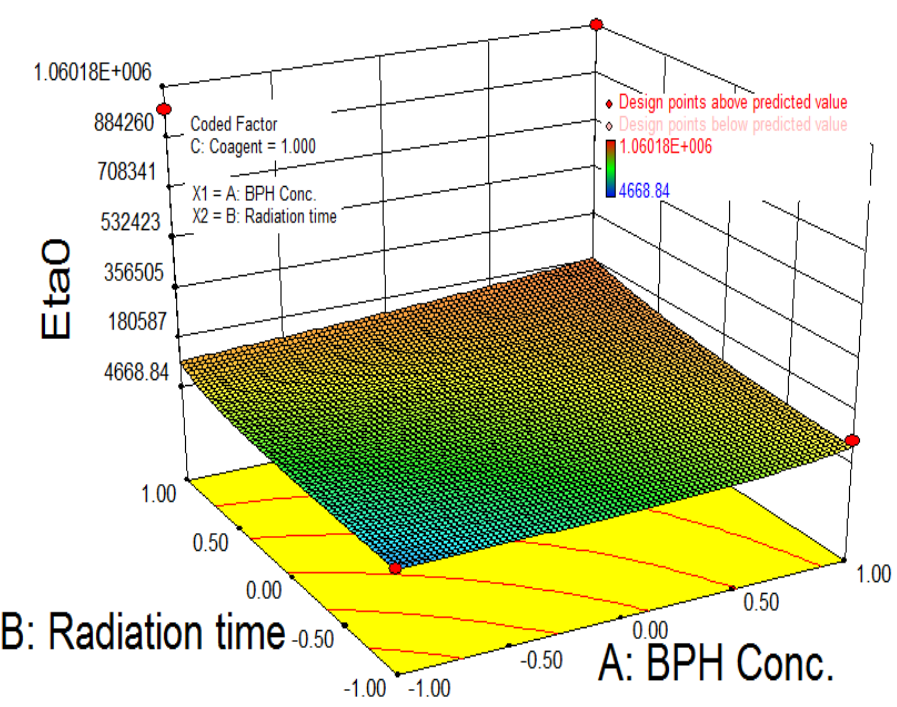

b

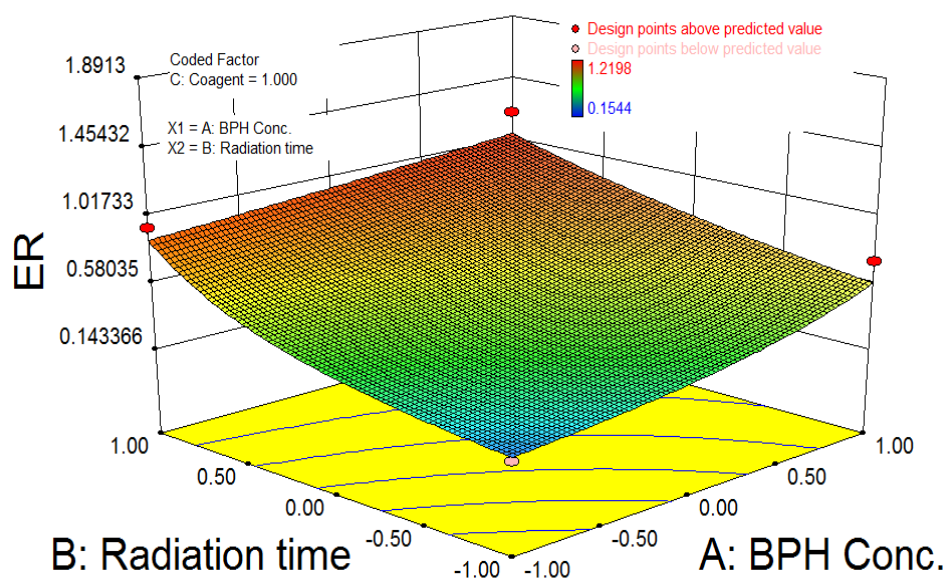

d

Figure 4: 3D interaction plots for $\eta_{0}$ and ER of the runs prepared without (a and c) and with coagent (b and d)

\subsection{GEL CONTENT MEASUREMENTS}

The percentage of gel for each run was determined as per section 2, and the values are summarized in Table 2. Among runs prepared with no coagent, only run 4, with the highest $\mathrm{BPH}$ concentration and radiation time, contains crosslinked and insoluble structures. 
On the other hand, among runs prepared with coagent, only run 5, which is clearly degraded (see Table 2 and the discussion in section 3.1), has no gel in its structure. Increasing BPH concentration from run 5 to 6 leads to formation of about $2.5 \%$ gel in the modified PP. Formation of gel might be undesirable for certain applications. However, considering the significant improvement in rheological properties of run 6, 2.5\% gel is still an acceptable margin. In contrast to runs 5 and 6 , increasing BPH concentration at high radiation time (runs with coagent), causes formation of less gel in the samples (see runs 7 and 8). This trend has also been observed for batch photomodification in the presence of coagent, with the same mechanism responsible [24]. When BPH concentration increases, more radicals will be formed; consequently, the number of PP macroradicals undergoing degradation ( $\beta$-scission) increases (since the TMPTA concentration is not sufficiently high to prevent degradation). Thus, the number of PP macroradicals which do not have TMPTA in their structure increases. The probability of reaction between PP macroradicals (which are protected by TMPTA) and products of the $\beta$-scission reaction (PP with a double bond at its chain end or secondary PP macroradicals), or tertiary PP macroradicals (PP macroradicals prior to $\beta$-scission), increases. These reactions encourage formation of long chain branches rather than crosslinked networks. Hence, although $\eta_{0}$ and ER of these runs have significantly increased, the gel content is relatively low. At low radiation time, the concentration of radicals is not large enough due to insufficient progress in the extent of reaction. Thus, as BPH concentration increases, gel content also increases.

Increasing radiation time (see runs 6 and 8, or run 2) causes formation of a significantly larger amount of gel in the runs. This is due to an increases in the probability of hydrogen abstraction from the chains that are already long chain branched. This leads to formation of connected network structures. This is again in agreement with results from batch modification. Details of these reactions and the related mechanisms can be found in reference [24].

Same as for the rheological indicators, an empirical model was used to show the significant variables and interactions affecting the gel content of the runs (Table 3). The model confirms that $\mathrm{BPH}$ concentration, radiation time and presence of coagent all have a significant and 
positive effect on the gel content of the runs. The 3 -factor interaction (ABC) was also found to be significant. Figure 5 shows representative factor interactions in two 3D surface plots. Figure 5a shows the $\mathrm{AB}$ interaction when coagent was not used, and Figure $5 \mathrm{~b}$ is for the case when coagent was used. Again, the maximum gel content was reached when coagent was used. The above mentioned trends with coagent can also be observed in Figure 5b (at low radiation time, an increase in $\mathrm{BPH}$ concentration leads to a higher gel content, while at high radiation time a lower amount of gel results).

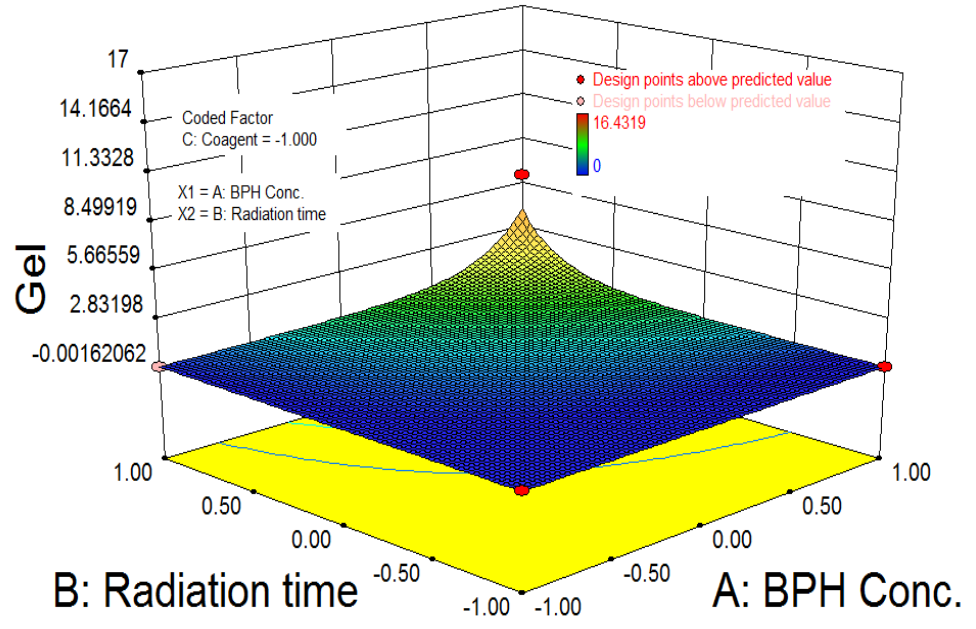

a

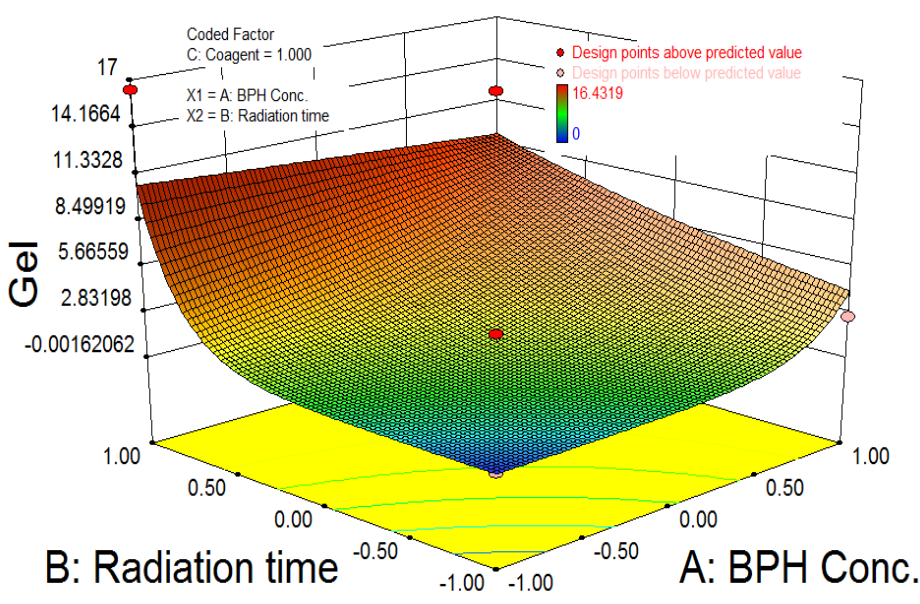

b

Figure 5: 3D interaction plots for gel content a) without coagent b) with coagent

\subsection{CONDITIONS THAT RESULT IN LONG CHAIN BRANCHED PP WITH MINIMUM GEL CONTENT}

In order to find the conditions that maximize LCB while having the lowest gel content during the continuous modification of PP, contour plots were constructed for $\eta_{0}, \lambda, n, E R$ and gel content. Certain specifications were set for each response in order to locate the (optimal)

processing window. Table 4 shows such specifications that lead to optimal processing conditions. The specifications in Table 4 are such that the corresponding shear thinning, zero shear viscosity, relaxation time and ER value ranges of the modified PP will be significantly 
greater than those of the parent PP. Hence, utilizing these specifications, formation of branches can clearly be confirmed. In addition, by setting gel content to be less than $5 \%$, we tried to identify conditions within the experimental range that result in the minimum gel content (therefore, overall optimal conditions that yield a balance between LCB and gel content).

Table 4: Response variables and their specs for locating the optimal processing condition region

\begin{tabular}{cc}
\hline Response & Specifications \\
\hline $\mathrm{n}$ & $<0.48$ \\
\hline$\eta_{0}(\mathrm{~Pa} . \mathrm{s})$ & $>10,000$ \\
\hline$\lambda(\mathrm{s})$ & $>3$ \\
\hline $\mathrm{ER}$ & $>0.4$ \\
\hline Gel content $(\%)$ & $<5$ \\
\hline
\end{tabular}

The contour plots corresponding to these processing specs are shown for runs without and with coagent (TMPTA) in Figure $6 \mathrm{a}$ and b, respectively. The optimal processing window, which satisfies these limits for the response variables (specs of Table 4), is shaded in black. 


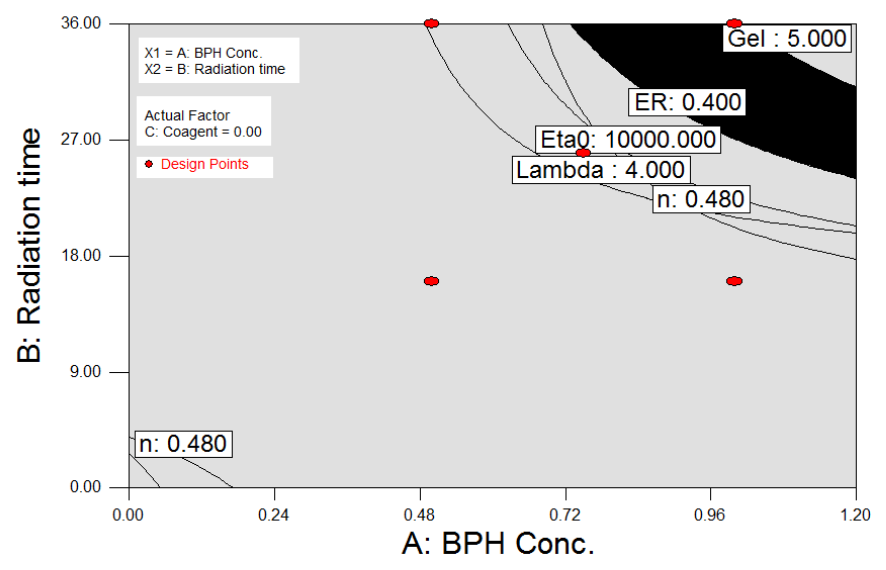

a

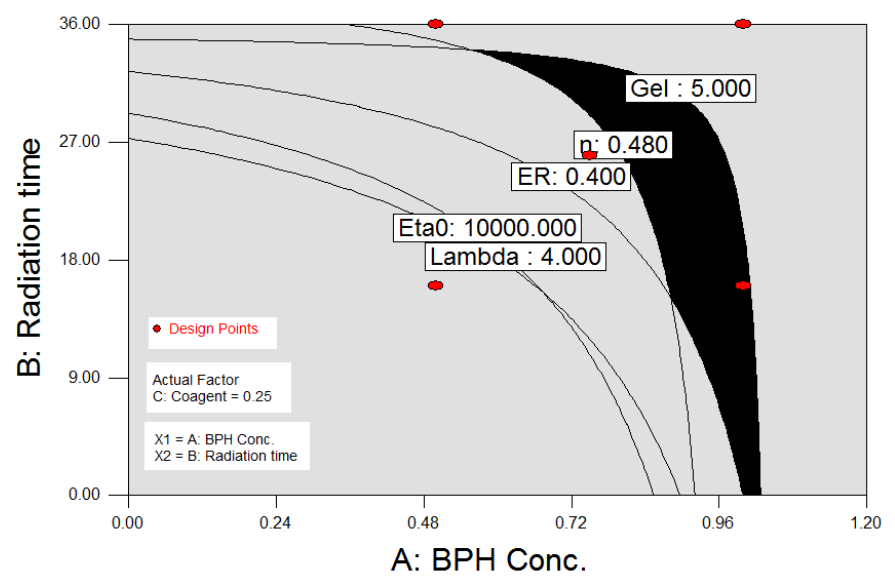

$\mathrm{b}$

Figure 6: Conditions that yield high degree of LCB without significant gel content a) without TMPTA, b) with TMPTA

\subsection{GPC MEASUREMENTS}

GPC measurements were selectively conducted on runs 4, 6, 8 and the parent PP. These runs were chosen because they manifest clear evidence for LCB in their rheological measurements (see Table 2 and the discussion in section 3.1). Run 4 was the only run prepared without coagent that shows indications of formation of long chain branches. Runs 6 and 8 were both prepared using TMPTA, and both runs show indications of formation of LCBs. The gel content of run 6 was exceptionally low, while its rheological properties confirmed formation of a large amount of LCBs. Run 8 was chosen since it had the largest $\eta_{0}, \lambda$ and ER, and the lowest $\mathrm{n}$ value among all runs. Figure $7 \mathrm{a}$ and $\mathrm{b}$ show the MWD and $\log [\eta]-\log \mathrm{M}$, respectively, for these selected runs.

The MWD of all these runs shows presence of a tail at the high MW ranges (Figure 7a). For runs 6 and 8, this tail at high molecular weights has almost formed a shoulder, which confirms formation of larger molecules due to LCB.

Figure $7 \mathrm{~b}$ shows $\log [\eta]$ vs. $\log \mathrm{M}$. Deviations of the modified runs from the linear parent PP towards lower $[\eta]$ values indicate formation of LCBs. All three modified runs deviate from the linear PP at molecular weights above $450 \mathrm{~kg} / \mathrm{mol}$. Since all three modified runs deviate 
almost equally from the linear PP, the weight average number of long chain branches $\left(\mathrm{B}_{\mathrm{w}}\right)$ in these polymers was found to be the same and around 0.26 branches per molecule. This value was calculated by solving ( 6 and 7 .

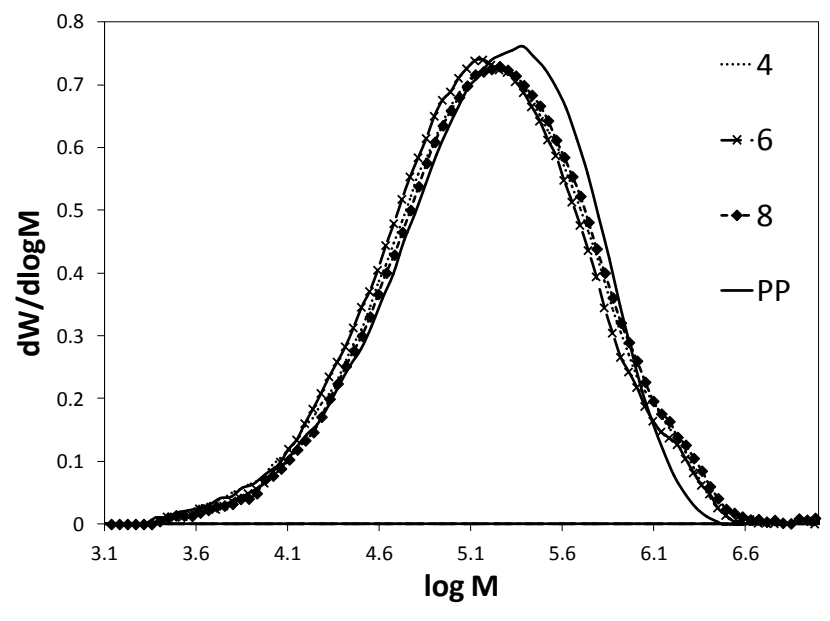

a

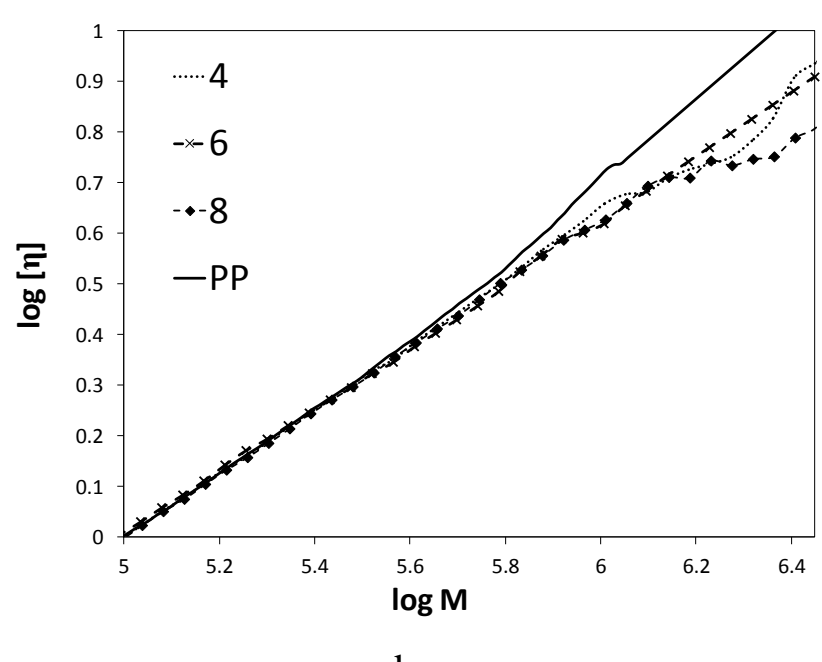

b

Figure 7: MWDs and $\log [\eta]$ vs. $\log \mathrm{M}$ for runs 4, 6, 8 and the parent PP

Using the MWD, number average molecular weight $\left(\bar{M}_{n}\right)$, weight average molecular weight $\left(\bar{M}_{w}\right)$, z average molecular weight $\left(\bar{M}_{z}\right)$ and polydispersity index (PDI) values were calculated and the results are summarized in Table 5. As expected from Figure 7a, all modified runs have greater $\bar{M}_{w}$ and significantly larger $\bar{M}_{z}$ than the parent PP. Also, an increase in the PDI of the samples shows that the MWD has become broader due to the presence of long chain branches. As expected, run 8 has the largest $\bar{M}_{w}, \bar{M}_{z}$ and the broadest MWD, which is in agreement with the rheological measurements (Table 2).

Table 5: Molecular weight averages and PDI of the selected radiated runs along with the parent PP

\begin{tabular}{cccccccc}
\hline $\begin{array}{c}\text { Sample } \\
\text { ID }\end{array}$ & $\begin{array}{c}\text { BPH } \\
\text { content } \\
(\mathbf{w t} \%)\end{array}$ & $\begin{array}{c}\text { Radiation } \\
\text { time (s) }\end{array}$ & $\begin{array}{c}\text { Coagent } \\
\text { content } \\
(\mathbf{w t} \%)\end{array}$ & $\begin{array}{c}\overline{\boldsymbol{M}}_{\boldsymbol{n}} \\
(\mathbf{k g} / \mathbf{m o l})\end{array}$ & $\begin{array}{c}\overline{\boldsymbol{M}}_{\boldsymbol{w}} \\
(\mathbf{k g} / \mathbf{m o l})\end{array}$ & $\begin{array}{c}\overline{\boldsymbol{M}}_{\boldsymbol{z}} \\
(\mathbf{k g} / \mathbf{m o l})\end{array}$ & PDI \\
\hline 4 & 1 & 36 & 0 & 70 & 288 & 551 & 4.1 \\
\hline 6 & 1 & 16 & 0.25 & 69 & 279 & 641 & 4.0 \\
\hline 8 & 1 & 36 & 0.25 & 73 & 339 & 1,229 & 4.7 \\
\hline $\mathrm{PP}$ & 0 & 0 & 0 & 73 & 271 & 274 & 3.7 \\
\hline
\end{tabular}




\section{CONCLUDING REMARKS}

A new design was proposed for the continuous modification of PP using UV radiation. Essentially, the batch modification of PP was scaled up to the continuous processing design for commercialization purposes. The modification was carried out on PP strands after melt mixing in a twin screw extruder. In order to achieve the radiation time needed for formation of long chain branches (LCBs), the extruded PP strands were folded between two rollers several times. Radiation was carried out between rollers and the modified strands were collected on a winder. The effect of BPH concentration, radiation time and coagent concentration on the rheological behaviour and gel content were studied. Zero shear viscosity $\eta_{0}$, relaxation time $\lambda$, shear thinning index $\mathrm{n}$, rheological polydispersity indices (PI, ER and ModSep) and the gel content were determined, and empirical models (based on a statistical experimental design) were fit to each of these response variables. Using these models, optimal processing conditions that result in long chain branching rather than crosslinking in PP were obtained.

\section{ACKNOWLEDGEMENTS}

The authors gratefully acknowledge financial support from the Natural Sciences and Engineering Research Council (NSERC) of Canada, and the Canada Research Chair (CRC) program.

\section{REFERENCES}

[1] C. Tzoganakis, Adv. Polym. Technol., 9, 321-330 (1989).

[2] G. Moad, Progress in Polymer Science, 24, 81-142 (1999).

[3] S. Bettini and J. Agnelli, J Appl Polym Sci, 85, 2706-2717 (2002). 
[4] E.V. Prut and A.N. Zelenetskii, Russian Chemical Reviews, 70, 65-79 (2001).

[5] H. Azizi and I. Ghasemi, Polym. Test., 23, 137-143 (2004).

[6] C. Tzoganakis, J. Vlachopoulos, and A. Hamielec, Polymer Engineering \& Science, 28, 170-180 (1988).

[7] G. He and C. Tzoganakis, Polymer Engineering \& Science, 51, 151-157 (2011).

[8] D. Graebling, Macromolecules, 35, 4602-4610 (2002).

[9] R. Lagendijk, A. Hogt, A. Buijtenhuijs, and A. Gotsis, Polymer, 42, 10035-10043 (2001).

[10] X. Wang, C. Tzoganakis, and G.L. Rempel, J Appl Polym Sci, 61, 1395-1404 (1996).

[11] P. Sardashti, C. Tzoganakis, M.A. Polak, and A. Penlidis, Macromolecular Reaction Engineering, 8, 100-111 (2014).

[12] M. Rätzsch, M. Arnold, E. Borsig, H. Bucka, and N. Reichelt, Progress in Polymer Science, 27, 1195-1282 (2002).

[13] Y. Amintowlieh, C. Tzoganakis, S. G. Hatzikiriakos and Alexander Penlidis, Polymer Degradation and Stability, 104, 1-10 (2014).

[14] Y. Amintowlieh, C. Tzoganakis, and A. Penlidis, J Appl Polym Sci, 131, 41021-41032 (2014).

[15] P. Zamotaev, E. Shibirin, and Z. Nogellova, Polym. Degrad. Stab., 47, 93-107 (1995).

[16] G. He, Y. Huang, and Q. Yang, Journal of Wuhan University of Technology-Mater. Sci. Ed., 28, 798-803 (2013).

[17] J. Tian, W. Yu, and C. Zhou, Polymer, 47, 7962-7969 (2006).

[18] R. Shroff and H. Mavridis, J Appl Polym Sci, 57, 1605-1626 (1995).

[19] J.A. Langston, R.H. Colby, T.M. Chung, F. Shimizu, T. Suzuki, and M. Aoki, Macromolecules, 40, 2712-2720 (2007).

[20] M. Scorah, R. Dhib, and A. Penlidis, Encyclopedia of Polymer Processing (ECHP), S.Lee, Ed., Taylor and Francis, New York, 251 (2005). 
[21] B. Krause, D. Voigt, A. Lederer, D. Auhl, and H. Munstedt, Journal of Chromatography A, 1056, 217-222 (2004).

[22] B.H. Zimm and W.H. Stockmayer, J. Chem. Phys., 17, 1301-1314 (1949).

[23] Y. Amintowlieh, Rheological Modification of Polypropyelene by Incorporation of Long Chain Branches Using UV Radiation, PhD Thesis, Department of Chemical Engineering, University of Waterloo (2014).

[24] Y. Amintowlieh, C. Tzoganakis, and A. Penlidis, Polymer-Plastics Technology and Engineering, manuscript length 45 pgs, accepted in Nov. 2014 (2015). 